Z. Klin. Chem. Klin. Biochem.

12. Jg. 1974 , S. $440-443$

\title{
Zur Methodik der Phenolrot-Bestimmung im Plasma
}

\author{
Von G. Heimann \\ Aus der Kinderklinik (Direktor: Prof. Dr. E. Gladtke) der Univerșität Köln
}

(Eingegangen am 5. Juni/31. Juli 1974)

\begin{abstract}
Die bisher bekannten Methoden zur Phenolrot-(Phenosulfonphthalein)-bestimmung im Plasma verlangen hämolysefreie Analysenproben. Bestimmungen aus dem Kapillarblut im Mikrolitermaßstab waren dadurch bisher nicht möglich. Mit der beschriebenen Methode gelingt es, nach Verdünnung des Plasmas mit Essigsäure den Farbstoff aus seiner Albuminbindung vollständig zu verdrängen. Nach anschließender Präzipitation der Proteine einschließlich des Hämoglobins wird der Farbumschlag durch Zugabe alkalischêr Äquivalente im Überschuß quantitativ erfaßt. Plasmatrübungen oder Plasmaeigenfarbe (Hyperbilirubinämie) beeinflussen die Extinktionsmessung nicht.
\end{abstract}

\section{Methodology for the determination of phenol red in plasma}

The measurement of the concentration of phenol red (phenolsulfonphthaleine) in plasma is disturbed by hemolysis. Estimations in capillary blood on a microscale are therefore impossible. In the new method phenol red is displaced from its albumin-binding by acetic acid. After precipitation of plasma proteins and hemoglobin the color change can be measured completely by adding alkaline equivalents in excess. The extinction measurement is not influenced by turbidity or hyperbilirubinemia.

Der als pH-Indikator bekannte Farbstoff Phenolrot (Phenolsulfonphthalein) ist für die funktionelle Nierendiagnostik geeignet, da er bei der Nierenpassage vorwiegend tubulär sezerniert wird $(1,2,3)$. Zur Beurteilung der tubulären Sekretionsleistung der Niere kann die innerhalb einer bestimmten Zeit ausgeschiedene Farbstoffmenge in Relation zur verabfolgten Dosis gemessen werden $(4,5)$.

Auf die Nachteile dieser Methoden ist wegen inkonstanter Diurese und notwendiger Katheterisierung mit Harnblasenspülung wiederholt hingewiesen worden $(6,7,8)$. Bei Kindern ist das Verhältnis zwischen Restharnvolumen aus dem Nierenbecken und den Ureteren und der gewonnenen Harnmenge noch ungünstiger als bei Erwachsenen. Auch Harnabflußstörungen, Atonie der Ureteren oder vesico-ureteraler Reflux verfälschen den Phenolrotausscheidungstest. Diese Fehlermöglichkeiten entfallen, wenn anstatt dieser Verfahren die Zeitclearance mit Hilfe der Phenolrotelimination aus dem Plasma bestimmt wird $(9,10,11)$.

Bisher wurden verschiedene Phenolrot-Plasma-Tests angegeben $(12,13,14)$. Diese Methoden benötigen mindestens $1 \mathrm{ml}$ Plasma oder Serum. Sie liefern nur bei absolut hämolysefreien Analyseproben exakte Ergebnisse, weil sich die Absorptionsmaxima von Hämoglobin und Phenolrot überschneiden. Da zur Bestimmung der Zeitclearance mit Hilfe der kinetischen Standardgrößen (Eliminationshalbwertzeit und totale Clearance) mehrere Blutentnahmen notwendig sind, war die Anwendung der bisher bekannten Phenolrot-Plasmä-Tests in der Pädiatrie erheblich eingeschränkt. Besonders fehlte die Möglichkeit der Bestimmung im Mikrolitermaßstab aus dem Kapillarblut, da dabei unterschiedlich starke Hämolyseraten zu erwarten sind.

Es lag daher nahe, eine Methode zu entwickeln, die folgenden Anforderungen genügt:

1. Die Konzentration von Phenolrot im Plasma muß quantitativ erfaßt werden.

2. Die bei der Entnahme von Kapillarblut zu erwartenden unterschiedlichen Hämolyseraten dürfen keinen Einfluß auf das Meßergebnis haben.

3. Die Methode muß unabhängig von der Eigenfarbe des Plasmas (z. B. Bilirubin) und von Plasmatrübungen sein.

\section{Material und Methodik}

Phenolrot ${ }^{1}$ ) lag als Natriumsalz gelöst in dèst. Wasser in einer Konzentration von $16,93 \mathrm{mmol} / 1$ vor. Als Reagenzien wurden $\mathrm{NaOH}(6,75 \mathrm{~mol} / 1)$, Essigsäure in Konzentrationen von

$1,66 \mathrm{~mol} / 1,2,49 \mathrm{~mol} / 1,4,16 \mathrm{~mol} / 1$ und Trichloressigsäure $3 \mathrm{~mol} / 1$ verwendet.

Die Extinktionsmessungen erfolgten bei $546 \mathrm{~nm}$ in einer Mikrodurchflußküvette (Schichtdicke $10 \mathrm{~mm}$, Spaltbreite $1 \times 4 \mathrm{~mm}$,

1) Phenolrot (Phenolsulfonphthalein) Fa. Merck, Darmstadt, gekennzeichnet als 0,6\%ige Lösung, $10 \mathrm{ml}$ Ampulle.

Z. Klin. Chem. Klin. Biochem. / 12. Jahrg. 1974 / Heft 10 
Füllvolumen $200 \mu 1)$. Für die in-vitro-Versuche wurden Mischplasmen unterschiedlicher Plasmaproteinkonzentration (41-83 g/1), sowie eine standardisierte Hämoglobinlösung (1,53 mmol/1) benutzt. Mit einer Bilirubinstandardlösung (Bilirubin Control, Dade) wurde der Einfluß der Plasmaeigenfarbe auf die Messung bestimmt.

\section{Methodik}

1. $100 \mu 1$ Plasma werden zunächst mit $400 \mu 1$ Essigsäure $(4,15 \mathrm{~mol} / \mathrm{l})$ verdünnt.

2. Zur Enteiweißung werden $200 \mu \mathrm{l}$ Trichloressigsäure ( $3 \mathrm{~mol} / \mathrm{l}$ ) hinzugegeben, 2 Minuten geschüttelt und 10 Minuten bei Raumtemperatur stehen gelassen.

3. Nach Zentrifugation für 2 Minuten bei $12000 \mathrm{U} . / \mathrm{min}$ werden $500 \mu \mathrm{l}$ als Aliquot des Überstandes zu $250 \mu \mathrm{l} \mathrm{NaOH}$ $(6,75 \mathrm{~mol} / \mathrm{l})$ pipettiert. Das Endvolumen beträgt $750 \mu \mathrm{l}$.

4. Nach kurzem Mischen kann die Extinktionsmessung bei $546 \mathrm{~nm}$ gegen Wasser als Leerwert erfolgen. Das Mitfüren eines Plasmaleerwertes ist nicht erforderlich. Bei wiederholten Kontrollen ergab sich stets gegen Wasser die Extinktion 0.

Zur Berechnung der Phenolrotkonzentration wird die gemessene Extinktion mit dem Eichfaktor multipliziert. Dieser ergibt sich als Produkt des durch die Eichkurve ermittelten Faktors $\mathrm{f}=\frac{\mathrm{C}}{\mathrm{E}}=12,2 \mathrm{mit}$ dem methodisch bedingten Verdünnungsfaktor 1,4. Dieser kommt dadurch zustande, daß nur $500 \mu 1$ des Überstandes zu $250 \mu 1 \mathrm{NaOH}(6,75 \mathrm{~mol} / \mathrm{l})$ gegeben werden. Das $\mathrm{Be}-$ zugsvolumen beträgt aber $700 \mu l$. Der Fehler, der mit der Präzipitation der Proteine durch Änderung des Gesamtvolumens eintritt, ist wesentlich kleiner als der methodische und kann daher vernachlässigt werden. Der Eichfaktor beträgt $170,8 \mu \mathrm{mol} / 1$ (oder $60,6 \mathrm{mg} / \mathrm{l}$ ).

\section{Bestimmung des zufälligen Fehlers}

Durch 20fach-Analysen wurde bei verschiedenen Phenolrotkonzentrationen der methodische Fehler bestimmt. Die statistische Auswertung ergab einen Variationskoeffizienten von $2,27 \%$.

\section{Ergebnisse}

Die Aufstellung einer Eichkurve erfolgte durch 1:100 Verdünnung der Phenolrot-Standardlösung (16,93 mmol/1) mit destilliertem Wasser oder physiologischer $\mathrm{NaCl}$ Lösung. Abbildung 1 zeigt, daß diese Eichkurve von Phenolrot in wäßriger Lösung innerhalb des geprüften Konzentrationsbereichs dem Lambert-Beer'schen Gesetz folgt.

\section{Wiederfindungsversuche}

Die Bestimmung der Wiederfindungsrate wurde mit verschiedenen Mischplasmen unterschiedlicher Phenolrotkonzentrationen $(8,46-67,72 \mu \mathrm{mol} / 1)$ nach Fällung der Plasmaproteine in Abhängigkeit von der vorherigen Verdünnung des Plasmas geprüft. Nach Zugabe von $400 \mu$ l physiologischer $\mathrm{NaCl}$-Lösung zu $100 \mu$ l Plasma waren weniger als $80 \%$ des zugefuihrten Farbstoffs nachweisbar (Abb. 1, Tab. 1). Bei Verdünnung mit Essigsäure der Konzentrationen 1,66 mol/1, 2,49 mol/1 und 4,16 mol/1 betrugen die Wiederfindungsraten im Mittel $82,5 \%, 87,9 \%$ und $97,8 \%$.

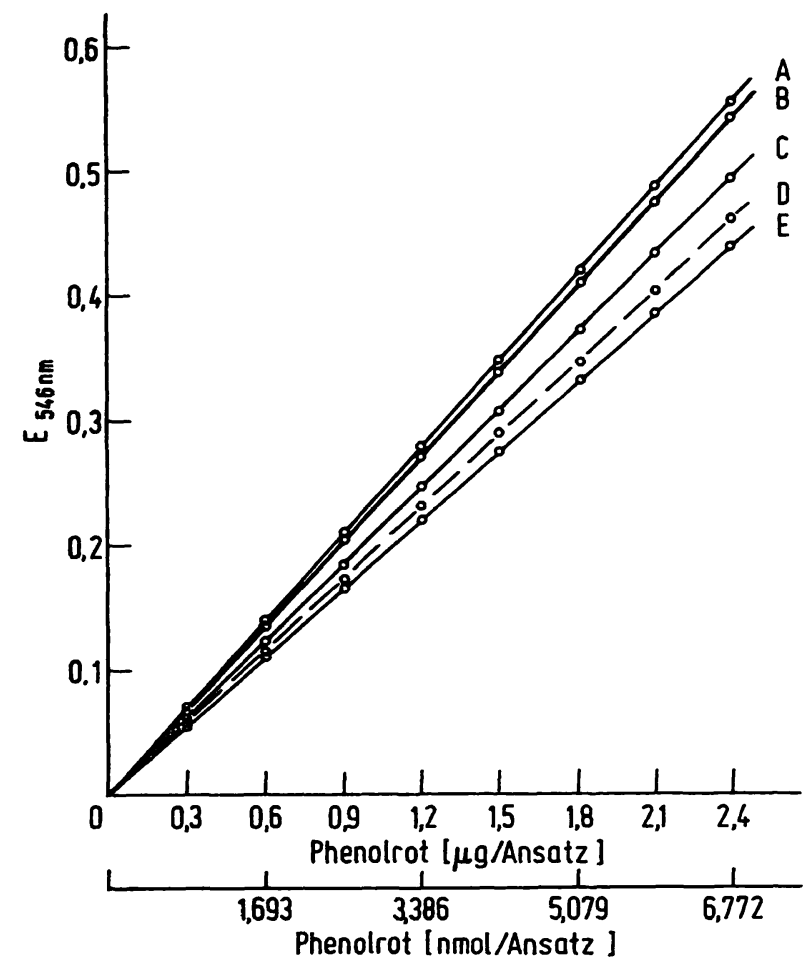

Abb. 1. Eichkurve und Wiederfindungsexperimente:

Die Gerade A stellt die Eichkurve für Phenolrot in wäßriger Lösung dar; die Geraden B, C, D sind Eichkurven, die aus Mischplasmen nach Verdünnung mit Essigsäure ( $B=4,16 \mathrm{~mol} / \mathrm{l}, \mathrm{C}=2,49 \mathrm{~mol} / \mathrm{l}, \mathrm{D}=1,66 \mathrm{~mol} / \mathrm{l}$ ) erhalten werden, die Gerade $E$ nach Verdünnung mit physiologischer $\mathrm{NaCl}$-Lösung.

Tab. 1. Wiederfindungsversuche:

Dargestellt sind die Mittelwerte $\overline{\mathbf{x}}$ und die Standardabweichungen (s) aus jeweils $10 \mathrm{fach}$-Bestimmungen. In der ersten Spalte sind die vorgegebenen Phenolrotkonzentrationen in $\mu \mathrm{mol} / 1$ angegeben. In den folgenden Spalten sind die Phenolrotkonzentrationen angegeben, die nach Verdünnung mit Essigsäure 4,16 mol/1 (B), 2,49 mol/1 (C), 1,66 mol/1 (D) und physiologischer NaCl-Lösung (E) erhalten werden.

\begin{tabular}{|c|c|c|c|c|c|c|c|c|}
\hline \multirow{2}{*}{$\mu \mathrm{mol} / \mathbf{1}$} & \multicolumn{2}{|l|}{ B } & \multicolumn{2}{|l|}{ C } & \multicolumn{2}{|l|}{ D } & \multicolumn{2}{|l|}{ E } \\
\hline & $\overline{\mathbf{x}}$ & $\pm s$ & $\overline{\mathbf{x}}$ & $\pm s$ & $\overline{\mathbf{x}}$ & $\pm s$ & $\overline{\mathbf{x}}$ & $\pm s$ \\
\hline $\begin{array}{r}8,46 \\
16,93 \\
25,40 \\
33,8 \mathrm{t} \\
42,33 \\
50,79 \\
59,26 \\
67,72\end{array}$ & $\begin{array}{r}8,46 \\
16,65 \\
25,11 \\
33,58 \\
41,76 \\
49,95 \\
58,69 \\
67,16\end{array}$ & $\begin{array}{l}0,08 \\
0,20 \\
0,23 \\
0,31 \\
0,48 \\
0,45 \\
0,79 \\
0,71\end{array}$ & $\begin{array}{r}7,34 \\
15,24 \\
22,29 \\
30,19 \\
37,25 \\
44,87 \\
52,49 \\
60,10\end{array}$ & $\begin{array}{l}0,08 \\
0,11 \\
0,20 \\
0,51 \\
0,23 \\
0,42 \\
0,51 \\
0,68\end{array}$ & $\begin{array}{r}7,05 \\
14,39 \\
21,16 \\
28,78 \\
35,84 \\
42,33 \\
49,95 \\
56,72\end{array}$ & $\begin{array}{l}0,06 \\
0,03 \\
0,06 \\
0,20 \\
0,23 \\
0,37 \\
0,23 \\
0,51\end{array}$ & $\begin{array}{r}6,77 \\
13,83 \\
20,60 \\
27,37 \\
33,86 \\
40,07 \\
46,56 \\
53,61\end{array}$ & $\begin{array}{l}0,08 \\
0,06 \\
0,14 \\
0,17 \\
0,20 \\
0,17 \\
0,34 \\
0,45\end{array}$ \\
\hline
\end{tabular}


Wurde anstatt der Essigsäure mit äquimolaren Mengen Salzsäure verdünnt, so konnten in vergleichenden Versuchsserien immer nur etwa $80 \%$ des zugeführten Farbstoffs bestimmt werden.

\section{Abhängigkeit von der Plasmaproteinkonzentration}

Der Einfluß unterschiedlicher Plasmaproteinkonzentrationen auf die Wiederfindungsrate wurde mit verschiedenen Mischplasmen der Proteinkonzentrationen 41, 62 und $83 \mathrm{~g} / 1$ geprüft. Bei Phenolrotkonzentrationen von 8,46-67,72 $\mu \mathrm{mol} / 1$ ergaben sich beim Vergleich der einzelnen Plasmen untereinander kaum meßbare Differenzen, die wesentlich kleiner als der methodische Fehler waren.

\section{Einfluß der Hämolyse}

Zum Nachweis des Einflusses der Hämolyse wurden Plasmaproben unterschiedlicher Phenolrotkonzentration mit steigenden Mengen einer standardisierten Hämoglobinlösung (1,53 mmol/1) versetzt. Tabelle 2 zeigt, daß sich bei Hämoglobinkonzentrationen von $30,7-$ $246,1 \mu \mathrm{mol} / 1$ keine Unterschiede gegenüber den hämolysefreien Plasmaproben ergaben. Entsprechende Versuchsserien ohne Zusatz von Phenolrot führten zu keinen meßbaren Extinktionen.

\section{Abhängigkeit von Plasmaeigenfarbe und Trübungen}

Plasmaeigenfarbe wie bei Hyperbilirubinämie oder Plasmatrübungen stören die bisher bekannten Phenolrotbestimmungen im Plasma oder Serum insbesondere bei niedrigen Farbstoffkonzentrationen. Versuchsserien mit steigenden Plasmabilirubinkonzentrationen von 17$85 \mu \mathrm{mol} / \mathrm{l}$ (entsprechend etwa 10-50 mg/1 Plasma) zeigten keinen Einfluß auf die Farbstoffwiederfindung (Tab. 3). Plasmatrübungen wurden nach Ansäuern mit Essigsäure (4,15 mol/1) und anschließender EnteiweiBung beseitigt, die Extinktionsmessung bei $546 \mathrm{~nm}$ wurde nicht gestört. Wird die gewonnene Blutprobe bald nach der Entnahme zentrifugiert, 'so kann das Plasma etwa 14 Tage lang bei $4^{\circ} \mathrm{C}$ aufbewahrt werden, ohne daß ein Farbstoffverlust eintritt. Die Zentrifuga-

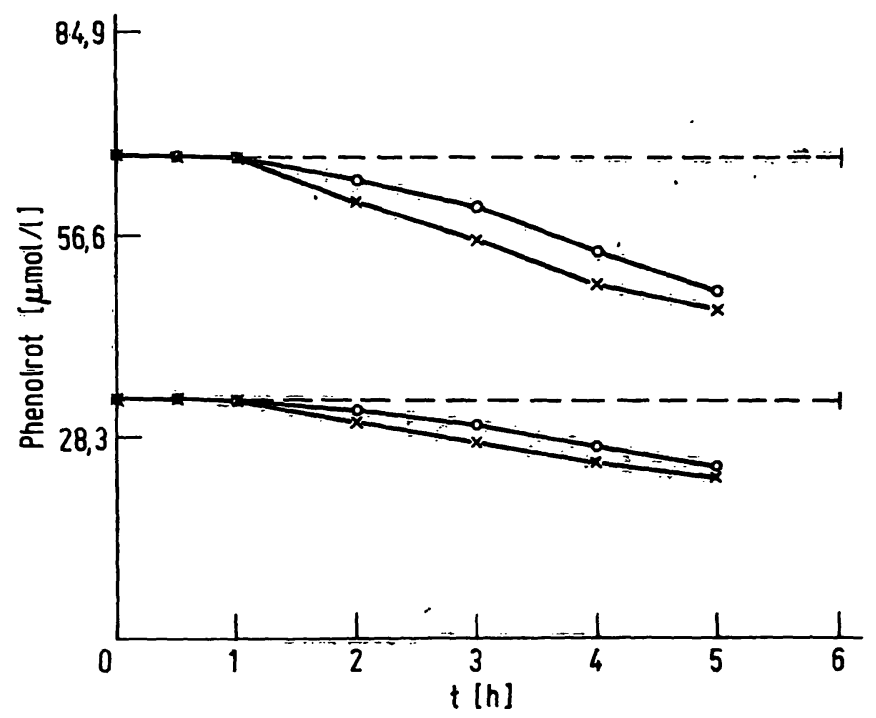

Abb. 2. Wiederfindung von Phenolrot aus Vollblut in Abhängigkeit von der Zeit der Aufbewahrung nach der Blutent: nahme und der Temperatur $\left(0-0=+4^{\circ} \mathrm{C}, x-x=\right.$ $+20^{\circ} \mathrm{C}$ ). Angegeben sind die Mittelwerte aus $4 \mathrm{fach}-\mathrm{Be}$ stimmungen.

Tab. 2. Abhängigkeit von der Hämolyse:

Die Mittelwerte der gemessenen Phenolrotkonzentrationen in $\mu \mathrm{mol} / 1$ und die Standardabweichung (s) sind angegeben bei unterschiedlichem Hämoglobingehalt des Plasmas (10fach-Bestimmungen).

Hämoglobinkonzentration

\begin{tabular}{|c|c|c|c|c|c|c|c|c|}
\hline & \multicolumn{2}{|c|}{$30,77 \mu \mathrm{mol} / 1$} & \multicolumn{2}{|c|}{$61,54 \mu \mathrm{mol} / 1$} & \multicolumn{2}{|c|}{$123,0 \mu \mathrm{mol} / 1$} & \multicolumn{2}{|c|}{$246,1 \mu \mathrm{mol} / 1$} \\
\hline & $\overline{\mathbf{x}}$ & $\pm s$ & $\overline{\mathbf{x}}$ & $\pm s$ & $\overline{\mathbf{x}}$ & $\pm s$ & $\overline{\mathbf{x}}$ & $\pm s$ \\
\hline $\begin{array}{r}8,46 \\
16,93 \\
25,40 \\
33,86 \\
42,33 \\
50,79 \\
59,26 \\
67,72\end{array}$ & $\begin{array}{r}8,46 \\
16,93 \\
25,11 \\
33,86 \\
42,05 \\
50,23 \\
58,98 \\
67,72\end{array}$ & $\begin{array}{l}0,06 \\
0,17 \\
0,11 \\
0,42 \\
0,34 \\
0,45 \\
0,34 \\
0,51\end{array}$ & $\begin{array}{r}8,75 \\
16,93 \\
24,83 \\
33,58 \\
41,76 \\
50,51 \\
58,41 \\
68,01\end{array}$ & $\begin{array}{l}0,08 \\
0,20 \\
0,14 \\
0,42 \\
0,42 \\
0,14 \\
0,31 \\
0,14\end{array}$ & $\begin{array}{r}8,18 \\
16,65 \\
25,11 \\
34,14 \\
42,05 \\
50,79 \\
58,69 \\
67,72\end{array}$ & $\begin{array}{l}0,06 \\
0,20 \\
0,20 \\
0,40 \\
0,45 \\
0,45 \\
0,42 \\
0,54\end{array}$ & $\begin{array}{r}8,46 \\
16,93 \\
24,83 \\
33,58 \\
42,33 \\
51,07 \\
58,98 \\
68,01\end{array}$ & $\begin{array}{l}0,03 \\
0,17 \\
0,23 \\
0,31 \\
0,40 \\
0,14 \\
0,48 \\
0,31\end{array}$ \\
\hline
\end{tabular}

Tab. 3. Abhängigkeit von der Bilirubinkonzentration:

Die Mittelwerte in $\mu \mathrm{mol} / 1$ und die Standardabweichung sind angegeben in Abhängigkeit von der Gesamtbilirubinkonzentration des Plasmas (10fach-Bestimmungen).

Bilirubinkonzentration

\begin{tabular}{lllllllllll}
- & & \multicolumn{2}{l}{$17,12 \mu \mathrm{mol} / 1$} & & $34,24 \mu \mathrm{mol} / 1$ & \multicolumn{2}{c}{$51,37 \mu \mathrm{mol} / 1$} & \multicolumn{3}{c}{$85,62 \mu \mathrm{mol} / 1$} \\
$\overline{\mathrm{x}}$ & $\pm \mathrm{s}$ & $\overline{\mathrm{x}}$ & $\pm \mathrm{s}$ & $\overline{\mathrm{x}}$ & $\pm \mathrm{s}$ & $\overline{\mathrm{x}}$ & $\pm \mathrm{s}$ & $\overline{\mathrm{x}}$ & $\pm \mathrm{s}$ \\
\hline 17,02 & 0,11 & 16,93 & 0,23 & 17,16 & 0,20 & 17,02 & 0,11 & 16,79 & 0,11 \\
\hline
\end{tabular}


tion des Blutes sollte jedoch nicht länger als 1 Stunde nach der Entnahme erfolgen. Danach fallt die Wiederfindungsrate deutlich ab und beträgt nach 5 Stunden bei Raumtemperatur nur noch etwa $70 \%$ der ursprünglichen Menge (Abb. 2). Diese Befunde stimmen mit Untersuchungen überein, die in vivo die Penetration von Phenolrot in die Erythrocy ten ausschließen, in-vitro jedoch nach längerem Stehen Schwundraten von 5\%/ Stunde nachweisen $(15,1)$.

\section{Diskussion}

Bei der bisher bekannten Methode zur Phenolrot-Bestimmung aus Plasma beschränken sich Höffler et al (12) auf eine einfache Alkalisierung von Plasma mit Natronlauge. Wesentliche Nachteile dieser Technik sind neben großen Schwankungen der Eigenextinktion des Plasmas inkonstante niedrige Wiederfindungsraten. Die Ursache dafür sind nach Fabricius \& Marowski (13) $\mathrm{pH}$-Verschiebungen durch den sinkenden $\mathrm{CO}_{2}$-Gehalt des Plasmas während des Untersuchungszeitraumes. Deshalb wurde von ihnen eine Methode angegeben, in der die Differenz der Extinktionen nach Zugabe von saurem ( $\mathrm{pH} 3,4)$ und alkalischem (pH 11,5) Phosphatpuffer gemessen wird. Die Wiederfindungsrate wird dadurch auf über $97 \%$ verbessert, vergleichbare Ergebnisse werden auch von Helger \& Wendt (14) mitgeteilt. Alle diese Techniken sind jedoch nur bei klaren, nicht hämolytischen Analyseproben anwendbar.

Nach Schwartzkopff (16) und eigenen Untersuchungen (17) ist es möglich, 3-4 mg Phenolrot pro kg Körpergewicht zu injizieren, ohne daß dadurch die Selbstdepression der Clearance erreicht wird und die kinetischen Größen der Zeitclearance verfälscht werden. Die hohe Farbstoffdosierung hat den Vorteil, daß Plasmakonzentrationen erreicht werden, die Bestimmungen aus kleinen Kapillarblutmengen ermöglichen. Wesentliche Voraussetzung dafür ist jedoch, daß der Einfluß der dabei auftretenden Hämolyse eliminiert werden

\section{Literatur}

1. Goldring, E., Clarke, R. W. \& Smith, H. W. (1936), J. Clin. Invest. 15, 221-228.

2. Grollman, A. (1925), J. Biol. Chem. 64, 141-160.

3. Smith, W. W. \& Smith, H. W. (1938), J. Biol. Chem. 124, 107-113.

4. Möller, J. \& Bedö, A. (1952), Ärztl. Wochenschr. 48, 1125-1128.

5. Stave, U. (1956), Z. Kinderheilk. 77, 554-562.

6. Heidelmann, G., Koch, E. \& Haake, M. (1956), Verh. Deut. Ges. Inn. Med. 62, 621-623.

7. Heintz, R. (1956), Deut. Med. Wochenschr. 82, 20442048.

8. Mertz, D. P. \& Sarre, H. (1962), Klin. Wochenschr. 40, 692-696.

9. Hand, G. (1953), Medizinische 25, 953-955.

10. Czok, G. (1963), Klin. Wochenschr. 41, 243-244.

11. Gault, M. H. (1966), Canad. Med. Ass. 94, 61-67. kann. Versucht man, Hämoglobin mit den Plasmaproteinen zu fällen, so wird ein Teil des an Albumin gebundenen Farbstoffs mit präzipitiert, die Wiederfindungsrate ist inkonstant und liegt bei etwa $80 \%$. Durch vorherige Verdünnung des Plasmas mit Essigsäure kann sie verbessert werden. Bei Verwendung von Essigsäure $(4,15 \mathrm{~mol} / \mathrm{l})$ beträgt die Wiederfindungsrate $97,8 \%$. Dieser Effekt ist nach Zugabe äquimolarer Mengen $\mathrm{HCl}$ nicht zu beobachten. Danach gelingt die Verdrängung der schwachen Säure Phenolsulfonphthalein aus ihrer Bindung an Albumin nicht durch Erhöhung der $\mathrm{H}^{+}$-Ionen-Konzentration. Von anderen eiweißgebundenen Farbstoffen, wie z. B. Bilirubin, ist bekannt, daß sie durch Zugabe von Anionen (Natrium-Acetat, NatriumBenzoat, Coffein und Theophyllin) aus ihrer Proteinbindung verdrängt werden können (18). Auch Phenolrot scheint durch eine definierte Konzentration von Acetat-Ionen aus seiner Albuminbindung vollständig freigesetzt $z u$ werden. Erst dann ist zu erwarten, daß durch Zugabe alkalischer Äquivalente im Überschuß der charakteristische Farbumschlag quantitativ erfaßt wird.

Versuche von Ochwadt \& Pitts (19), Phenolrot durch Aceton zu extrahieren, gelingen nur bei hämolysefreiem Plasma. Schon bei geringer Hämolyse gehen Farbkomplexe in Lösung, die nicht mehr mit Trichloressigsäure präzipitierbar sind und die Extinktionsmessung erheblich verfälschen. Ähnliche Beobachtungen konnten auch bei Verwendung von Methanol und Äthanol gemacht werden.

Die hier beschriebene Methode der Phenolrotbestimmung aus dem Plasma verlangt gegenüber den bisher üblichen Methoden durch die vollständige Enteiweißung einen zusätzlichen Analysenschritt. Der damit verbundene geringe Mehraufwand erscheint dadurch gerechtfertigt, daß bei nahezu vollständiger Farbstoffwiederfindung Bestimmungen im Mikrolitermaßstab auch aus stärker hämolytischem Kapillarblut möglich sind.

12. Höffler, D., Offermann, G. \& Frenz, H. (1968), Deut. Med. Wochenschr. 93, 397-402.

13. Fabricius, W. \& Marowski, B. (1970), diese Z. 8, 431-433.

14. Helger, R. \& Wendt, F. (1970), Klin. Wochenschr. 48 , 1006-1008.

15. Smith, H. W., Goldring, W. \& Chaisis, H. (1938), J. Clin. Invest. 17, 263-278.

16. Schwartzkopff, W. (1969), Z. Gesamte Exp. Med. 150, 120-138.

17. Heimann, G. \& Twarock-Bergt, B. vorgetragen: 10. Arbeitstagung für Pädiatrische Forschung Marburg 18. 19. April 1974.

18. zitiert aus: Richterich, R. (1971), Klinische Chemie, 3. Aufl. S. Karger, Basel, S. 455.

19. Ochwadt, B. K. \& Pitts, R. F. (1956), Amer. J. Physiol. $187,318-322$.
Dr. G. Heimann

Universitätskinderklinik Köln $5 \mathrm{Köln} 41$

Josef-Stelzmann-Straße 9 